\title{
Lidil
}

Revue de linguistique et de didactique des langues

$50 \mid 2014$

Variation stylistique et diversité des contextes de socialisation

\section{Discours sociolinguistiques et discours profanes face à la variation stylistique dans la prononciation du français}

Sociolinguistic and Ordinary Discourses about Phonostylistic Variation in French

\section{Maria Candea}

\section{OpenEdition}

\section{Journals}

Édition électronique

URL : http://journals.openedition.org/lidil/3557

DOI : 10.4000/lidil.3557

ISSN : 1960-6052

\section{Éditeur}

UGA Éditions/Université Grenoble Alpes

\section{Édition imprimée}

Date de publication : 15 décembre 2014

Pagination : 45-61

ISBN : 978-2-84310-287-5

ISSN : $1146-6480$

\section{Référence électronique}

Maria Candea, « Discours sociolinguistiques et discours profanes face à la variation stylistique dans la prononciation du français », Lidil [En ligne], 50 | 2014, mis en ligne le 15 juin 2016, consulté le 19 avril 2019. URL : http://journals.openedition.org/lidil/3557 ; DOI : 10.4000/lidil.3557 


\title{
Discours sociolinguistiques et discours profanes face à la variation stylistique dans la prononciation du français
}

\author{
Maria Candea*
}

\begin{abstract}
RÉSUMÉ
Cet article propose une réflexion sur les relations entre les discours épilinguistiques profanes et ceux des spécialistes en sociophonétique au sujet des prononciations du français. Les résultats obtenus grâce à une enquête socioperceptive menée auprès de 68 auditeurs francophones de France, de tous âges, sont d'abord résumés et ensuite confrontés aux tendances qui se dégagent des publications scientifiques contemporaines sur la variation phonostylistique.

Il apparait que, si globalement les sociophonéticiens déconstruisent plus que les «profanes » les stéréotypes stigmatisants pesant sur les locuteurs socialement minorés, leur démarche reste encore inachevée. En revanche, ils utilisent des catégories beaucoup moins sophistiquées que les auditeurs ordinaires pour caractériser les styles de ceux qui bénéficient d'un grand prestige social. L'article plaide pour un renouvèlement des recherches dans le sens de la diversification des descripteurs de la variation phonostylistique et d'une focalisation plus rigoureuse sur l'agentivité des individus, quel que soit leur profil social.
\end{abstract}

\section{ABSTRACT}

This paper aims to investigate the relations between epilinguistic ordinary discourses and those of experts in sociophonetics about the French pronunciation. The results obtained through a socioperceptual survey based on 68 French-speaking listeners from France, of all ages, are first summarized and then compared with trends in contemporary scientific publications about phono-stylistic variation.

It appears that sociophoneticians generally deconstruct more than "ordinary people" the stereotypes about lower-class speakers, but their approach still remains incomplete. However, experts use much fewer categories than our listeners to characterize the phono-style of speakers with high social prestige. The conclusion argues for a renewal of

* Université Sorbonne Nouvelle-Paris 3, CLESTHIA. 
the research through a diversification of the descriptors used for characterize the phono-stylistic variation and a more clear focus on the agency of individuals, regardless of their social profile.

La tradition sociolinguistique puise dans les travaux pionniers de Labov (1972) deux idées majeures : premièrement le fait que tout locuteur est pluristyle selon le degré de surveillance de ses productions linguistiques, même si cela peut se manifester à des degrés divers selon les situations et les classes sociales, et deuxièmement le fait que les styles de prononciation sont aisément reproductibles de manière expérimentale selon qu'on demande aux gens de lire une liste de paires minimales, un texte, ou bien selon qu'on les place en situation d'entretien formel ou de conversation spontanée. Ce paradigme a été adopté tel quel ou avec des ajustements par de nombreux sociolinguistes qui étudient la prononciation du français, avec parfois des focalisations particulières sur certains aspects : la variation en fonction de l'âge et du prestige acquis (Duez, 1991; Fagyal, 1995), de l'origine régionale (Durand, Laks \& Lyche 2009), de la classe ou de l'affiliation sociale (notamment Mettas, 1979; Jamin, 2005 ; Hansen, 2000 ; Fagyal, 2010 ; Lekha-Lemarchand, 2011), du genre (Bento, 1998), etc.

Une cinquantaine d'années plus tard, la notion de «style de prononciation» a été profondément redéfinie notamment grâce aux méthodes ethnographiques d'investigation : Eckert (2010) ou Mendoza (2008) mettent en lumière des variations stylistiques très fines dans la prononciation selon les enjeux - évolutifs - de la situation, les postures identitaires en interaction ou les stratégies argumentatives.

La variété des points de vue des chercheurs et des corpus étudiés font que nous disposons à présent d'un grand nombre d'études détaillées sur la variation stylistique dans la prononciation en français, que ce soit au sujet des locuteurs que Bourdieu (1982) appellerait «légitimes», ceux qui imposent leur parole sur le marché linguistique d'une société, ou sur les locuteurs «illégitimes », ceux dont la parole est socialement minorée. Les connaissances produites par ces études sont, comme toutes connaissances, socialement et historiquement situées et, compte tenu du sujet qui suscite facilement des discours épilinguistiques, nous pouvons nous attendre à ce qu'il y ait une certaine porosité entre les discours des sociolinguistes et ceux des locuteurs non spécialistes de la langue.

Comme le notait déjà, il y a une trentaine d'années, Tajfel (1981, p. 223) au sujet des identités sociales, les processus parallèles d'unification et de diversification sont plus rapides que jamais dans un contexte 
où des groupes humains très divers entrent en communication les uns avec les autres à une très vaste échelle ; cela doit avoir fortement contribué à configurer les discours sur autrui, ceux des sociolinguistes comme ceux de tout francophone. Confronter ces deux types de discours permettra d'apporter des éléments de réponse aux questions suivantes : peut-on repérer quelques convergences et divergences entre les discours savants produits par la sociolinguistique devenue institutionnelle et les discours dits profanes? L'observation des discours profanes peut-elle continuer à nourrir les études futures en sociolinguistique?

La première partie de cet article présentera une analyse rapide du sentiment linguistique profane (Paveau, 2009) au sujet de la prononciation, tel qu'il résulte d'une récente étude que j'ai pu mener à l'aide d'un groupe d'étudiant-e-s du master de Lettres modernes de 1'Université Sorbonne Nouvelle, auprès d'un échantillon ad hoc. La seconde partie résumera, en miroir, les tendances des études sociolinguistiques sur la variation stylistique dans la prononciation du français, pour pouvoir proposer, en conclusion, une comparaison entre les deux types de discours et quelques pistes de recherches.

\section{Susciter des discours épilinguistiques auprès de non linguistes}

Pour documenter des discours épilinguistiques actuels en France, j'ai construit un protocole assez simple d'enquête par questionnaire, que j'ai mis à l'épreuve en tant que pré-enquête auprès de deux personnes. La trentaine d'étudiants et étudiantes qui suivait mon séminaire de master de sociophonétique en 2012-2013 a eu comme consigne, pour le devoir, de recueillir des réactions évaluatives auprès de deux personnes de leur entourage (famille, voisins ou amis) au sujet de six extraits de parole enregistrée, d'une douzaine de secondes chacun. 68 personnes (hommes ou femmes, d'âges très variés, habitant dans différentes régions de France, profils sociaux divers) ont été sollicitées.

\subsection{Stimulus utilisés}

Les six échantillons de parole soumis à évaluation appartenaient à trois locuteurs ayant des métiers prestigieux liés à des compétences langagières réputées au-dessus de la moyenne (deux journalistes et une actrice) et à trois locuteurs ayant un statut moins valorisé et non lié à des compétences langagières particulières (deux lycéens, sans métier du fait de leur âge, et un ancien joueur de rugby). 
Les deux journalistes soumis à évaluation étaient Patrick Poivre d'Arvor (extrait d'un journal télévisé) et Catherine Ceylac (extrait d'interview de l'émission Thé ou Café); l'actrice était Fanny Ardant, en interview dans la même émission Thé ou Café. De nombreux auditeurs sollicités ont reconnu ces voix, notamment celle du présentateur vedette, ce qui était un effet prévisible. Cela permettait d'augmenter, le cas échéant, l'effet d'une profession valorisée en ajoutant celui de la notoriété d'une personne. Quant aux deux lycéens enregistrés, ils faisaient partie du groupe que j'ai été amenée à suivre durant ma dernière recherche de terrain (voir Candea, 2012a pour une présentation synthétique) et le commentateur de rugby était Richard Astre, s'exprimant dans un journal radiophonique podcasté à partir de France Info. Personne parmi les auditeurs ne pouvait reconnaitre ces trois dernières voix.

La perception des auditeurs et auditrices était volontairement amorcée $^{1}$ par les profils des personnes enregistrées, qui leur étaient indiqués ainsi : 1/ journaliste de télévision, présentateur de journal; 2/ ancien joueur de rugby, toulousain, consultant sportif sur une radio nationale; 3/ lycéen de Seine-Saint-Denis lors d'une préparation d'examen oral; 4/ lycéenne de Seine-Saint Denis lors d'un exercice de lecture; 5/ journaliste de télévision, lors d'un entretien avec une artiste; 6/ actrice, lors d'une interview à la télévision.

Pour donner une idée des caractéristiques de ces enregistrements, j'essaie de dresser ici une liste de traits de prononciation qui m'ont semblé susceptibles d'avoir une saillance perceptive.

Du côté des locuteurs connus, la prononciation de PPDA et celle de C. Ceylac contenaient différentes marques caractéristiques du phonostyle journalistique : emphase prosodique, allongements vocaliques parfois remarquables, épithèses fricatives particulièrement longues (Candea, 2012b), et chez Ceylac deux «e d'appui» (ou épithèses vocaliques, Carton, 1999; Candea, 2002). La prononciation de F. Ardant contenait des voyelles nasales très ouvertes («intempéries» prononcé [ãtãpeRi]), des épithèses fricatives particulièrement longues, une insistance inhabituelle sur certaines consonnes occlusives $/ \mathrm{k}, \mathrm{t} /$ prononcées avec beaucoup d'énergie, des allongements vocaliques emphatiques; elle parlait en voix soufflée durant tout cet extrait, sur le ton de la confidence-séduction.

1. Pour la notion d'amorçage (priming) dans les protocoles expérimentaux de perception de la parole, se rapporter par exemple à Signoret (2010). 
Du côté des autres locuteurs, le lycéen produisait un contour saillant montant-descendant ultra-rapide que Lehka-Lemarchand (2011) considère comme une caractéristique emblématique de l' «accent de banlieue», des /t/ affriqués au contact avec /j/, des /a/ postérieurs, un «euh» d'hésitation et un allongement du connecteur «ben». La lycéenne prononçait des /t/ et /d/ fortement affriqués, la nasale /ã/ proche du [õ] assez fermé et postérieur, deux /R/ pharyngaux ainsi que des liaisons considérées comme très fréquentes non réalisées, comme par exemple «quand\#il». Enfin, le commentateur de rugby produisait une prononciation globalement marquée par l'accent du sud-ouest : schwas finaux prononcés, nasales allongées et partiellement dénasalisées.

Tous les extraits contenaient un lexique courant plutôt soutenu mais des prononciations qu'on peut qualifier de «non standard», assez typantes, susceptibles d'être perçues et identifiées par les auditeurs sollicités.

\subsection{Questions posées}

Le questionnaire fourni aux étudiants comportait cinq questions sur le caractère «standard» ou «non standard» de la prononciation, sur les sons perçus comme prononcés de manière remarquable et sur l'appréciation globale de l'extrait de parole écouté. La dernière question demandait aux auditeurs de catégoriser chaque prononciation par rapport à leurs représentations du groupe dont faisait partie la personne enregistrée. Elle était formulée ainsi :

Pensez-vous que la journaliste que vous venez d'écouter a une façon très personnelle de parler, ou bien qu'elle prononce comme nombre de ses confrères? Explicitez, notamment si vous pouvez proposer des explications de sa façon de parler.

Les mots «journaliste» et «confrères » étaient remplacés respectivement par «lycéen» et «camarades» pour les extraits concernés, ou par «actrice» et «nombre d'autres actrices ».

Les auditeurs répondaient par écrit, de manière télégraphique, mais les étudiants-enquêteurs avaient comme consigne de noter également, dans la mesure du possible, les remarques produites oralement lors des écoutes, notamment si elles n'étaient pas consignées sur les feuilles des questionnaires.

Il ne serait pas pertinent de présenter ici des données quantifiées, homogénéisantes, dans la mesure où les enquêteurs et les lieux d'enquête étaient très divers, et la durée des entretiens n'était pas standar- 
disée (certains étudiants ont passé 20 à 25 minutes pour un entretien, d'autres ont passé une heure et demie). Je me contente donc de résumer ce qui me semble pertinent pour la réflexion sur la catégorisation des styles de parole.

\subsection{Tendances générales dans les réponses obtenues}

Nous nous attendions, au regard de la littérature de spécialité, à ce que la prononciation des locuteurs servant de modèles en matière de langage (journalistes et actrice) soit catégorisée comme standard et que leurs spécificités passent inaperçues, comme non marquées; de même nous nous attendions à ce que le fait d'avoir rendu saillantes les identités sociales des locuteurs entraine une stéréotypisation (Tajfel, 1981), chaque locuteur devenant un prototype de sa catégorie : le lycéen de banlieue parisienne, l'actrice, le présentateur télé, etc.

Or, il a été frappant de constater que les particularités de prononciation ont été globalement souvent mentionnées. Parfois dès la première question, de manière spontanée, parfois après la relance qui demandait aux auditeurs de focaliser leur attention sur la prononciation des voyelles et des consonnes (car certains avaient tendance à se focaliser plutôt sur le lexique, ou sur l'intonation).

Pour les trois locuteurs dont le statut n'induisait pas de compétences langagières particulières (l'ancien joueur de rugby et les deux lycéens), comme attendu, les auditeurs ont remarqué de nombreuses variantes de prononciation : nasales «du sud» pour le commentateur, nasales non conformes pour les lycéens («peinture» entendu comme «panture», /ã/ entendu comme / $/$ /), «e muets prononcés», accent trainant, $/ \mathrm{r} /$ «arabe ou guttural», /t/ «soufflé, chuintant, mouillé» (pour les occlusives affriquées), /a/ fermé, «tirant vers le 'o'», liaisons non faites, prononciation nasillarde...

Mais, contrairement à ce que l'on pouvait penser à priori, les métiers et la notoriété des trois autres locuteurs n'ont pas masqué leurs prononciations remarquables. Les auditeurs ont très souvent évoqué le rythme trainant de PPDA, le parler «soufflé» des deux journalistes et surtout de l'actrice, les nasales trop ouvertes de Fanny Ardant, les attaques occlusives particulièrement fortes de certains mots, les «e» rajoutés à la fin de certains mots. Les épithèses fricatives ont également été souvent perçues, notamment chez F. Ardant et C. Ceylac ${ }^{2}$. La journaliste en

2. PPDA en produisait une seule mais elle n'a jamais été clairement évoquée. 
produisait une exceptionnellement longue, suivie d'une épithèse vocalique après le mot «vous» (prononcé [vu:ç:ə]), et les auditeurs ont fait preuve d'une grande créativité graphique pour rendre compte de la prononciation perçue : «vous en sifflant, vousf, vouf, vouff, voufff, vousssssseuh, vouch, voufe, voufeu, vouchch...».

Les auditeurs n'ont pas mobilisé les catégories de «standard» et «non standard», et la demande portant sur l'appréciation subjective et globale des extraits écoutés n'a pas permis de départager les locuteurs aux métiers de la parole et les autres. En effet, les extraits les plus appréciés ont été ceux produits par C. Ceylac, la lycéenne et l'ancien sportif, tandis que les extraits les moins appréciés étaient ceux de PPDA (monocorde), du lycéen (inarticulé, agressif) et de F. Ardant qui a suscité tantôt des réactions de détestation (affectée, pompeuse, surannée, ampoulée, insupportable, pédante, égotique, castafiore...), tantôt d'enthousiasme (sublime, poétique, rêveuse, émouvante, aérienne...).

Globalement, les auditeurs se sont approprié les macro-catégories proposées dans le protocole et les ont reformulées en insistant soit sur les traits de prononciation des groupes sociaux (journalistes, ados, bobos, bourgeois, racaille, arabe, africain), soit sur les traits régionaux (parisien, pointu, sud-ouest, banlieue), soit les deux. En raison de la composition de l'échantillon interviewé (qui ne comportait ni lycéens, ni acteurs, ni journalistes), tous les auditeurs se sont positionnés comme étant extérieurs (outgroup) aux groupes d'affiliation des locuteurs écoutés, ce qui a enclenché un mécanisme d'évaluation intergroup et a facilité la formulation de critiques (Tajfel, 1981).

\subsection{Clivage entre les locuteurs en fonction de leur proximité avec les normes langagières prestigieuses}

En revanche, si l'on observe la grande variété des syntagmes produits pour «expliquer» les prononciations remarquables, en écart par rapport à une prononciation supposée neutre, un clivage subtil apparait entre nos deux catégories de locuteurs.

Pour les locuteurs «légitimes», les 'juges' formulent une grande diversité d'hypothèses faisant une large place à leurs choix stylistiques supposés, aux effets recherchés et en général à leur agentivité ou puissance d'agir («agency»: Butler, 1997). Les tendances générales sont résumées dans le tableau 1 : 


\begin{tabular}{|c|c|c|}
\hline Locuteur & Tendances des interprétations & Conclusion sur l'individuation \\
\hline PPDA & $\begin{array}{l}\text { - Adopte un ton monocorde ou mono- } \\
\text { tone parce qu'il se veut rassurant (il } \\
\text { parle d'une épidémie). } \\
\text { - Insiste sur certains mots pour } \\
\text { rendre l'information plus facile à } \\
\text { comprendre. } \\
\text { - Un style qui se veut efficace. }\end{array}$ & Style très personnel. \\
\hline C. Ceylac & $\begin{array}{l}\text { - Style d'interview culturelle } \\
\text { maniérée. } \\
\text { - Tente de construire une ambiance } \\
\text { chaleureuse, joviale, enjouée, sympa, } \\
\text { mondaine / essaie de mettre à l'aise } \\
\text { l'autre, de l'englober / se montre } \\
\text { faussement chaleureuse, hypocrite ou } \\
\text { pédante / adopte un ton snob, mièvre } \\
\text { ou précieux. } \\
\text { - On imagine presque sa gestuelle et } \\
\text { son sourire. }\end{array}$ & $\begin{array}{l}\text { Une façon bien à elle de } \\
\text { parler, parle d'une façon très } \\
\text { personnelle. }\end{array}$ \\
\hline F. Ardant & $\begin{array}{l}\text { - Rythme très saccadé mais personnel, } \\
\text { attaques fortes pour convaincre, } \\
\text { s'imposer ou conquérir l'autre / hésite } \\
\text { entre convaincre et se perdre dans son } \\
\text { monde par les mots. } \\
\text { - On a l'impression qu'elle essaie de } \\
\text { se faire désirer quand elle parle. } \\
\text { - A un ton de voix hautain et un } \\
\text { vocabulaire plutôt enrichi / ça la fait } \\
\text { passer pour une snob. } \\
\text { - Prononce comme d'autres actrices } \\
\text { le feraient au théâtre, pas dans une } \\
\text { interview. }\end{array}$ & $\begin{array}{l}\text { Une façon bien à elle de parler, } \\
\text { style très personnel. }\end{array}$ \\
\hline
\end{tabular}

Tableau 1. - Tendance des réponses au sujet des locuteurs dont la parole est réputée prestigieuse.

Ainsi, très souvent, malgré le fait qu'il ait une prononciation typique parisienne avec un phonostyle journalistique («formaté journal télé»), les auditeurs trouvent que PPDA a un style très personnel. Pour C. Ceylac, les réponses foisonnent d'adjectifs généralement convergents sur son style et son intention de créer une atmosphère spécifique. En ce qui concerne Fanny Ardant, il est plus difficile de construire un florilège représentatif dans la mesure où elle déclenche des réactions fortes positives ou négatives, mais les conclusions vont toutes vers le même consensus, très fort, d'une façon très personnelle de parler.

Pour les locuteurs minorés, les réponses récoltées montrent une tout autre tendance. Elles sont résumées dans le tableau 2. 


\begin{tabular}{|c|c|c|}
\hline Locuteur & Tendances des interprétations & Conclusion sur l'individuation \\
\hline $\begin{array}{l}\text { Ancien } \\
\text { sportif }\end{array}$ & $\begin{array}{l}\text { - Appuie sur les voyelles, il met des ' } g \text { ' } \\
\text { à la fin des mots. } \\
\text { - Il est dans le ton, on comprend tout } \\
\text { de suite que c'est du rugby. }\end{array}$ & $\begin{array}{l}\text { Parle exactement comme ses } \\
\text { confrères }^{\text {a. }} \\
\text { Parle façon sud-ouest et façon } \\
\text { rugby. }\end{array}$ \\
\hline $\begin{array}{l}\text { Lycéen de } \\
\text { Seine-Saint- } \\
\text { Denis }\end{array}$ & $\begin{array}{l}\text { - On sent l'envie de s'appliquer mais } \\
\text { avec beaucoup d'hésitation / on sent } \\
\text { qu'il doute et qu'il n'est pas assuré, } \\
\text { mais essaie de répondre malgré son } \\
\text { stress. } \\
\text { - Problème de l'agencement du } \\
\text { contenu : complique son discours à } \\
\text { cause de la construction. } \\
\text { - On dirait qu'il agresse son } \\
\text { interlocuteur. } \\
\text { - La fin des groupes syntaxiques } \\
\text { est relevée, comme s'il reprenait sa } \\
\text { respiration après une nage en apnée, } \\
\text { comme s'il était soulagé d'en avoir } \\
\text { fini / sa prononciation est un peu } \\
\text { mixée, pas très distincte, s'il fallait } \\
\text { l'écrire on a l'impression qu'il n'y } \\
\text { a pas de ponctuation / à la fin de ses } \\
\text { phrases, l'intonation monte au lieu de } \\
\text { descendre. } \\
\text { - Prononce comme beaucoup d'étran- } \\
\text { gers / sa parole n'est pas tout à fait } \\
\text { vulgaire, mais c'est limite / ça fait } \\
\text { vraiment racaille des cités. }\end{array}$ & $\begin{array}{l}\text { Parle comme ses camarades; } \\
\text { comme ses comparses; comme } \\
\text { une bonne partie de ses cama- } \\
\text { rades; comme la plupart des } \\
\text { jeunes des milieux populaires. } \\
\text { Pas personnel du tout, tous les } \\
\text { élèves de certains quartiers } \\
\text { parlent comme lui (banlieue). }\end{array}$ \\
\hline $\begin{array}{l}\text { Lycéenne de } \\
\text { Seine-Saint- } \\
\text { Denis }\end{array}$ & $\begin{array}{l}\text { - Ton très dynamique. } \\
\text { - Lecture banale, rien de particulier / } \\
\text { pas d'accent particulier, mais parle } \\
\text { trop vite et découpe ses phrases de } \\
\text { manière particulière. } \\
\text { - Accent africain, accent de banlieue } \\
\text { mais pas très fort. } \\
\text { - Moins de rythme rap que le lycéen } \\
\text { précédent. } \\
\text { - Elle a une voix joyeuse alors qu'elle } \\
\text { parle de la mort de Michael Jackson. } \\
\text { - Parle comme ses camarades, mais } \\
\text { fait un effort de diction dans ce travail. }\end{array}$ & $\begin{array}{l}\text { Parle comme ses camarades }{ }^{\mathrm{b}} \text {, } \\
\text { comme sa génération. } \\
\text { Comme beaucoup de gens quand } \\
\text { ils ne sont pas à l'aise pour lire } \\
\text { un texte en public. } \\
\text { Style assez habituel chez les } \\
\text { étrangers d'origine africaine. }\end{array}$ \\
\hline
\end{tabular}

a. Parmi les rares personnes ayant trouvé que le commentateur avait une façon personnelle de parler, une auditrice répondait ainsi : «Il a sa façon personnelle de commenter, on sent dans sa voix que le journalisme n'est pas son premier métier.» Autrement dit, selon cette auditrice, ce qui pouvait singulariser ce commentateur c'était le fait qu'il n'était pas journaliste, et qu'il ne présentait pas les traits de prononciation du corps de métier attendu.

b. Deux auditeurs ont préféré ne pas répondre à cette question en disant : «Il faudrait entendre d'autres jeunes en situation de lecture.»

\section{Tableau 2. - Tendance des réponses au sujet des locuteurs éloignés des variétés prestigieuses.}


Personne n'envisage la possibilité que les lycéens puissent avoir une façon personnelle de parler, et presque personne ne l'envisage pour l'ancien rugbyman. Un très large consensus se dégage là aussi, mais dans un sens contraire aux trois précédents : ils sont perçus comme prototypiques (comme ses camarades, comme ses confrères).

Au sujet du sportif devenu commentateur, les réponses étaient très courtes et je n'ai trouvé aucun verbe relevant du champ sémantique de l'intention, du vouloir ou de l'agentivité en général, si ce n'est quelques vagues «il appuie sur les voyelles» et absolument aucune trace de possibles choix stylistiques.

Pour le lycéen, chez qui pratiquement tous les auditeurs ont noté la présence de «ben», hésitant, en début d'énoncé, un seul commentaire a fait une place à son agentivité en matière de choix stylistique, formulé ainsi : «il fait sans doute des efforts, il ne parle pas comme avec ses copains ados ». Ce commentaire apparait donc comme tout à fait exceptionnel et peu représentatif car, par ailleurs, les seules remarques recueillies envisageaient le style ou les connotations de la prononciation du lycéen comme échappant entièrement à son contrôle et contraires à sa volonté (on dirait qu'il agresse, complique son discours). Sa subjectivité est souvent masquée par des tournures qui portent uniquement sur sa prononciation, décrite comme s'il s'agissait d'un phénomène sans sujet (la fin des groupes est relevée, sa prononciation est mixée, l'intonation monte, etc.).

En ce qui concerne la lycéenne, qui avait reçu des appréciations globalement plus positives pour son exercice de lecture à haute voix perçue le plus souvent comme «très dynamique », tous les auditeurs pensent que sa façon de lire n'a rien de personnel à l'exception de cinq qui évoquent tout de même ses efforts pour contrôler sa prononciation et se rapprocher $\mathrm{du}$ «standard».

Le clivage entre locuteurs légitimes et locuteurs illégitimes me semble assez bien résumé par une auditrice dans cette phrase produite spontanément après l'écoute du dernier extrait (Fanny Ardant) : «Ardant c'est quelqu'un qui aime la langue, on sent un plaisir de parler, de jouer avec la langue, contrairement au lycéen pour qui la langue est quelque chose de simplement fonctionnel. »

Si l'on tente de synthétiser les tendances observées en matière d'interprétation de la diversité des prononciations, on peut dire que lorsqu'on confronte des auditeurs profanes à des locuteurs exerçant des métiers prestigieux de la parole, ils relient rapidement les macrocatégories d'appartenance et les habitudes de prononciation (ici : bour- 
geois, journalistes, parisiens...), mais recherchent spontanément et systématiquement des traces d'individuation et des effets de style supposés intentionnels, qu'ils décrivent par une profusion de tournures.

En revanche, lorsqu'on confronte les mêmes auditeurs à la parole de locuteurs minorés, ceux-ci sont tout aussi rapidement rattachés à des macro-catégories d'appartenance (ici : Méridionaux, sportifs, jeunes de banlieue populaire...), mais il n'y a pratiquement jamais de recherche d'individuation ou de tentative d'identifier des effets de style supposés intentionnels. Les locuteurs minorés sont perçus comme prototypiques de leur groupe - envisagé comme homogène et inapte à la variation stylistique.

La macro-catégorisation unifiante suivie d'individuation pour les uns mais pas pour les autres est indépendante de l'empathie (ou totale absence d'empathie) avec les locuteurs écoutés.

\section{2. Études sur la variation stylistique dans la prononciation du français}

Intéressons-nous à présent aux tendances identifiables dans les publications académiques portant sur les prononciations. En 2000, dans un numéro de LINX consacré aux approches sociolinguistiques du plan phonique, Gadet comparait la profusion de termes pour désigner les profils des locuteurs et la remarquable pauvreté des termes pour les situations et les styles de parole associés. Elle mettait également en évidence ce que la notion de «relâchement» avait d'idéologique, pour caractériser à la fois la parole dite non surveillée (le style «relâché» étant une option pour les locuteurs avec un grand capital culturel) et la parole produite dans les milieux socialement minorés, supposée marquée par un «relâchement» articulatoire et musculaire inhérent, compatible avec les analyses de Bourdieu sur l'hexis corporelle. En dehors de ce paradigme assez réducteur, Gadet déplorait un manque flagrant de données et d'analyses.

Si l'on tente de faire un bilan des études disponibles une bonne dizaine d'années plus tard, on peut noter une évolution certaine depuis ce constat de quasi-absence formulé en 2000, mais leur nombre est toujours bien trop faible.

D'un côté, les recherches utilisant des protocoles pour observer la variation stylistique des locuteurs dominés restent rares et éparses (Trimaille, 2003 ; Jamin, 2005 ; Lambert, 2005 ; Buson, 2009; Lehka, 2011; Auzanneau, Leclère-Messebel \& Juillard, 2012) et cette question 
reste souvent périphérique au regard de l'étude, souvent suscitée par la demande sociale, des traits langagiers stigmatisants. Trimaille (2003) affirme vouloir "étayer empiriquement l'hypothèse selon laquelle les représentations et les attitudes ont une influence prépondérante dans la perception par les adultes de ce que Gueunier (2000) nomme une "frontière d'incommunicabilité" avec les enfants des cités», et LehkaLemarchand (2011) rappelle explicitement les idées reçues selon lesquelles les jeunes de couches populaires n'auraient aucune sensibilité aux variations diaphasiques. Auzanneau, Leclère-Messebel et Juillard (2012) évoquent la force de l'idée reçue selon laquelle les jeunes des milieux populaires en réinsertion après un parcours judiciaire sont incapables de variation stylistique, ainsi que l'hostilité à priori que rencontrent les conclusions contraires auprès des acteurs sociaux commanditaires des enquêtes. Par ailleurs, Lambert (2005) rend compte d'une expérience de jeux de rôles - interviews sur les marchés - menée dans un lycée professionnel, où le style de parole des élèves en rupture avec l'école changeait de manière radicale. Ce changement était tellement fort que, lorsqu'une des élèves a eu l'occasion de se réécouter une dizaine d'années plus tard, à la suite d'une rencontre fortuite avec la chercheuse, elle a refusé spontanément de croire qu'il s'agissait de son propre enregistrement et a pensé qu'il s'agissait d'une autre collègue.

D'un autre côté, les recherches portant sur des enregistrements de locuteurs dits «légitimes» s'intéressent certes souvent à la variation stylistique, mais celle-ci est appréhendée surtout à travers des oppositions entre lecture / conférence / entretien formel / conversation / narration, catégories peu nombreuses et fortement liées aux pratiques universitaires ou médiatiques (voir par exemple Mettas, 1979; Duez, 1991; Fagyal \& Moisset, 1999; Hansen 2000; Durand, Laks \& Lyche 2009³ ; Goldman, Auchlin \& Simon, 2009).

Autrement dit, malgré le postulat unanimement partagé en sociolinguistique selon lequel tous les locuteurs sont pluristyles, l'inventaire des études disponibles pour le français peut suggérer que nous aurions collectivement tendance à nous intéresser davantage aux variations stylistiques chez les locuteurs socialement valorisés. De plus, pour ces derniers, nous aurions tendance à appréhender la variation stylistique de la prononciation à travers une grille de pré-catégories fort rudimen-

3. Les données PFC (<www.projet-pfc.net $>$ ) prennent en compte la parole de locuteurs aux profils sociaux très divers. 
taire par rapport à celles qui ont cours par exemple dans les études stylistiques portant sur des textes littéraires. Il est très rare, par ailleurs, que des études visent à comparer les variations stylistiques d'individus appartenant à différents groupes sociaux, comme l'avait fait Labov (1972) dans son étude sur les /R/ à New York, où il interprétait la plus grande amplitude de variation entre la première occurrence de fourth floor et la seconde chez le groupe de classe moyenne comme le signe d'une plus grande insécurité linguistique.

\section{Discussion et conclusions}

Revenons à présent au point de départ de ces réflexions. En prenant en considération la doxa de la sociolinguistique, on s'attendrait à ce que les chercheurs et les chercheuses aillent à l'encontre des stéréotypes sociaux. Or, si nous observons les tendances générales dans les pratiques de recherche des sociolinguistes, dont je fais partie, à travers les publications disponibles, il est difficile de confirmer facilement une déconstruction nette de ces stéréotypes culturellement partagés, pour le moins dans le domaine de la sociophonétique.

Il est possible d'affirmer, selon moi, que pour l'étude des variations stylistiques des locuteurs socialement minorés, les sociolinguistes sont plutôt en avance sur les opinions profanes, mais cette avance est assez récente (Gadet, 2000) et encore bien trop timide. Il est vrai que les sociolinguistes défendent l'hypothèse selon laquelle tout locuteur est pluristyle et évitent activement les termes stigmatisants pour décrire les variantes socialement dévalorisées. Le relâchement articulatoire a plutôt fait place aux contraintes articulatoires et au comportement des sons en contact. Il n'en reste pas moins que les chercheurs sont encore trop rares à s'intéresser aux variations stylistiques et à la recherche d'expressivité, ou à l'exploitation adroite de l'iconicité des gestes articulatoires, et préfèrent le plus souvent s'en tenir à l'étude de «variables explicatives» comme l'âge, l'ethnicité, le genre, les langues d'héritage, la réussite scolaire (Fagyal, 2010) ou encore l'appartenance sociale ou la situation. Or, décrire les pratiques des locuteurs minorés uniquement à travers leurs affiliations de groupe ne permet pas de donner une place à leur agentivité.

Parallèlement, il me semble qu'au sujet de l'étude des variations stylistiques des locuteurs jouissant d'un grand prestige social, les sociolinguistes sont au contraire plutôt en retrait par rapport à l'opinion profane. La richesse foisonnante des hypothèses et nuances produites, 
après seulement quelques secondes de parole de deux journalistes et d'une actrice, par les auditeurs sollicités dans mon test de perception, contraste de manière saisissante avec la simplicité des catégories que nous, sociolinguistes en particulier sociophonéticiens, mobilisons généralement pour rendre compte des variations stylistiques, comme par exemple «lecture / entretien formel / conversation / interview politique», etc. La place importante accordée à la capacité des personnes écoutées pour agir subtilement sur autrui à travers la prononciation, construire une situation par la parole, marquer leur subjectivité, se distinguer, jouer avec le langage, s'oppose aux analyses en sociophonétique où nous parlons plutôt d'influence de la situation et de variation diaphasique, laissant peu de place à l'agentivité. Or, celle-ci s'invite parfois de manière imprévue même dans les études variationnistes classiques fondées sur des données sollicitées dans des situations standardisées. Par exemple, Hansen (2000) montre comment une simple tâche de lecture, présentée de manière identique à plusieurs personnes sollicitées, est investie différemment selon leurs niveaux de littéracie (bien que ce ne soit pas l'objectif premier de son étude). Ce constat est particulièrement flagrant lorsqu'on se penche sur les lectures de texte recueillies dans le cadre du protocole PFC (Phonologie du français contemporain) : selon les profils, les locuteurs et locutrices adoptent un style de lecture hyperarticulé, didactique ou enjoué, ou au contraire un style pressé, monologique, hypoarticulé et/ou monotone, ou produisent parfois une lecture laborieuse et appliquée qui révèle leur difficulté face à la tâche demandée. Ces observations sur la richesse de la variation stylistique remettent fortement en question l'hypothèse encore fort répandue selon laquelle il suffirait de donner le même texte et la même consigne à des personnes différentes pour obtenir «la même situation».

Les études ethnographiques se situent au pôle opposé, où aucune situation n'est identique à une autre. Par exemple, dans son ouvrage consacré aux gangs de jeunes filles dans une ville du nord de la Californie, Mendoza-Denton (2008, p. 113) évoque en détail un rendezvous très important qui a tourné court dès le rituel de salutation en raison d'un faux pas : l'interviewé avait initié l'échange en donnant son prénom (Manuel) prononcé [mæ:nyu:El] à l'anglaise, et la chercheuse a répondu en prononçant le sien (Norma) à l'espagnole, sans voyelle longue et avec un $/ \mathrm{r} /$ apical. Cette prononciation de la part d'une personne représentant l'université, dans un contexte où le choix de la langue fait l'objet de négociations subtiles, a été interprétée par Manuel, l'interviewé, comme une vexation, comme le signe que la chercheuse 
ne le croyait pas capable d'être suffisamment à l'aise en anglais. Pour ce type d'analyse, la macro-catégorie des salutations ne permet pas de rendre compte des contraintes sur la prononciation, et cela pose la question de la pertinence des catégories pour comparer les situations.

Trois directions de recherche me semblent à l'heure actuelle sousexploitées, au sujet du français.

Premièrement, il conviendrait certainement d'approfondir l'étude des contraintes qui pèsent sur la production des variantes de prononciation prestigieuses, car elles sont encore trop souvent présentées comme «neutres» et échappant à la fois aux contraintes articulatoires et à l'agentivité (recherche de distinction, hyper-articulation).

Deuxièmement, il serait nécessaire d'élargir et de multiplier les études portant véritablement sur la variation stylistique des locuteurs peu valorisés socialement, et veiller à poser le même type de questions sur les marqueurs sociolinguistiques valorisants et stigmatisants. Cela diminuerait le poids des stéréotypes sociaux qui nous incitent à réserver involontairement aux écrivains et aux personnages ayant une grande notoriété les questionnements stylistiques (voir Fagyal, 1995 sur Marguerite Duras), et aux groupes minorés les questionnements sur l'effet des langues en contact et des propriétés articulatoires des sons (Fagyal, $\left.2010^{4}\right)$. Cela permettrait de compléter les descriptions trop éparses et parcellaires sur les capacités de variation stylistique chez des personnes réputées peu habiles dans le maniement de la langue.

Troisièmement, il serait grand temps de dépasser les pré-catégories de genres discursifs trop simplifiants, trop vagues et trop peu nombreux pour affiner les analyses, en tirant profit des approches situées, ethnographiques et interactionnistes, complémentaires de l'approche par corrélations quantitatives. Cela ouvrirait un véritable chantier de réflexion sur la comparabilité des données.

Ajoutons enfin à ces trois pistes que, dans le sillage des hypothèses de Fonagy (1983) sur l'iconicité relativement stable de certains gestes articulatoires, il serait possible d'approfondir la complexité des relations entre ces éventuelles iconicités ou motivations articulatoires, les conventions sociales et la créativité stylistique individuelle.

4. Pourtant, après avoir montré que les macro-catégories prédéterminées échouent à rendre compte de manière satisfaisante des différences entre les collégiens étudiés, Fagyal (2010) admet la nécessité de se pencher sur le profil particulier d'un élève (un «passeur» interculturel) et sur ses stratégies stylistiques. 
Concevoir le langage comme une pratique complexe participant à la structuration sociale (et non reflétant simplement cette structure), une pratique dont les règles sont contestées et renégociées sans cesse, y compris dans le domaine phonétique, reste un défi à relever.

\section{RÉFÉRENCES BIBLIOGRAPHIQUES}

Auzanneau, Michelle, Leclère-Messebel, Malory \& Juillard, Caroline. (2012). Élaboration et théâtralisation de catégorisations sociolinguistiques en discours, dans une séance de formation continue. La catégorie «jeune» en question. Langage et société, 141, 47-69.

Bento, Margaret. (1998). Une étude sociophonétique des affriquées désonorisées en franco-québécois. Revue québécoise de linguistique, 26(1), 13-26.

Bourdieu, Pierre. (1982). Ce que parler veut dire : l'économie des échanges linguistiques. Paris : Fayard.

Butler, Judith. 2004 (1997). Le Pouvoir des mots. Politique du performatif. Paris : Éditions Amsterdam.

Buson, Laurence. (2009). Variation stylistique entre 5 et 11 ans et réseaux de socialisation scolaire : usages, représentations, acquisition et prise en compte éducative (Thèse de doctorat, Université Stendhal-Grenoble 3).

CAndeA, Maria. (2002). Le e d'appui parisien : statut actuel et progression. Actes des 24e JEP, 185-188.

Candea, Maria. (2012a). Départ : ZEP. Arrivée : Sciences-Po. Moyen de transport : le langage. Diversité. Ville-École-Intégration, 167, «Les jeunes des quartiers», 111-115.

CANDEA, Maria. (2012b). Au journal de RFI-chhh et dans d'autres émissions radiodiffusée-chhhs. Les épithèses fricatives. Le discours et la langue, 3, 136-149.

Duez, Danielle. (1991). La pause dans la parole de l'homme politique. Paris : éd. CNRS.

Durand, Jacques, Laks, Bernard \& Lyche, Chantal. (dir.), (2009). Phonologie, variation et accents du français. Paris-Londres : HermèsLavoisier éd.

ECKerT, Penelope. (2010). Affect, Sound Symbolism, and Variation. University of Pennsylvania Working Papers in Linguistics, 15(2), article 9.

FAGYAL, Zsuzsanna. (1995), Aspects phonostylistiques de la parole médiatisée lue et spontanée : âge, prestige, situation, style et rythme de parole de l'écrivain M. Duras (Thèse de doctorat, Université Paris 3).

FAgYal, Zsuzsanna. (2010). Accents de banlieue. Aspects prosodiques du français populaire en contact avec les langues de l'immigration. Paris : L'Harmattan. 
Fagyal, Zsuzsanna \& Moisset, Christiane. (1999). Sound Change and Articulatory Release: Where and Why Are High Vowels Devoiced in Parisian French? Actes ICPhS 99, 309-312.

FonAgy, Ivan. (1983). La vive voix. Essais de psycho-phonétique. Paris : Payot.

GADET, Françoise. (2000). Le terme «relâchement» en sociolinguistique. Linx. Revue des linguistes de l'université Paris X Nanterre, 42, «Approches sociolinguistiques du plan phonique», 11-20.

Goldman, Jean-Philippe, Auchlin, Antoine \& Simon Anne-Catherine. (2009). Discrimination de styles de parole par analyse prosodique semiautomatique. Dans H.-Y. Yoo \& É. Delais-Roussarie (dir.), Actes d'IDP (p. 207-221).

Hansen, Anita B. (2000). Le E caduc interconsonantique en tant que variable sociolinguistique. Une étude en région parisienne. Linx, 42, «Approches sociolinguistiques du plan phonique», 45-58.

Jamin, Michael. (2005). Sociolinguistic Variation in the Paris Suburbs (Thèse de doctorat, University of Kent at Canterbury).

Labov, William. 1976 (1972). Sociolinguistique. Paris : Éditions de Minuit.

LAMBERT, Patricia. (2005). Les répertoires plurilectaux de jeunes filles d'un lycée professionnel : une approche sociolinguistique ethnographique (Thèse de doctorat, Université Stendhal-Grenoble 3).

LEHKA-LEMARCHAND, Iryna. (2011). La stratification stylistique d'un indice prosodique de l'accent dit «de banlieue». Enquête auprès de jeunes Rouennais. Lidil, 44, 79-92.

Mendoza-Denton, Norma. (2008). Homegirls: Language and Cultural Practice among Latina Youth Gangs. Malden, MA : Blackwell Pub.

MetTas, Odette. (1979). La prononciation parisienne, aspects phoniques d'un sociolecte parisien (du Faubourg Saint-Germain à la Muette). Paris : SELAF.

Paveau, Marie-Anne. (2009). Quand Marie-Chantal dit merde : sentiment linguistique et normes perceptives dans la haute société. Dans G. Achard-Bayle \& M. Lecolle, Sentiment linguistique et discours spontanés sur le lexique (p. 41-63). Metz : CREM.

Signoret, Carine. (2010). Exploration des mécanismes non conscients de la perception de la parole : approches comportementales et électroencéphalographiques (Thèse de doctorat, Université Lyon 2).

TAjfel, Henri. (1981). Human Groups and Social Categories: Studies in Social Psychology. Cambridge : CUP Archive.

Trimaille, Cyril. (2003). Variations dans les pratiques langagières d'enfants et d'adolescents dans le cadre d'activités promues par un centre socio-culturel, et ailleurs. Dans J. Billiez \& D. de Robillard (dir.), Français : variations, représentations et pratiques (p. 113-161), Cahiers du français contemporain, 8. Lyon : ENS Éditions. 